\title{
MULTI-SCALE SEGMENTATION OF HIGH RESOLUTION REMOTE SENSING IMAGES BY INTEGRATING MULTIPLE FEATURES
}

\author{
Yanan Di ${ }^{\mathrm{a}}$, Gangwu Jiang ${ }^{\mathrm{b}}$, Libo Yan ${ }^{\mathrm{a}}$, Huijie Liu ${ }^{\mathrm{a}}$, Shulei Zheng ${ }^{\mathrm{a}}$ \\ ${ }^{a}$ R \& D Engineer, Beijing SatImage Information Technology Co.,Ltd, China \\ ${ }^{\mathrm{b}}$ Zhengzhou Institute of Surveying and Mapping,Zhengzhou, China
}

Chinese library classification number: P237

KEY WORDS: high resolution remote sensing images, multi-scale segmentation, canny edge detector, texture feature, Mumford Shah - standard, Kruskal algorithm

\begin{abstract}
:
Most of multi-scale segmentation algorithms are not aiming at high resolution remote sensing images and have difficulty to communicate and use layers' information. In view of them, we proposes a method of multi-scale segmentation of high resolution remote sensing images by integrating multiple features. First, Canny operator is used to extract edge information, and then band weighted distance function is built to obtain the edge weight. According to the criterion, the initial segmentation objects of color images can be gained by Kruskal minimum spanning tree algorithm. Finally segmentation images are got by the adaptive rule of Mumford -Shah region merging combination with spectral and texture information. The proposed method is evaluated precisely using analog images and ZY-3 satellite images through quantitative and qualitative analysis. The experimental results show that the multi-scale segmentation of high resolution remote sensing images by integrating multiple features outperformed the software eCognition fractal network evolution algorithm (highest-resolution network evolution that FNEA) on the accuracy and slightly inferior to FNEA on the efficiency.
\end{abstract}

\section{INTRODUCTION}

Image segmentation is the premise of object-oriented high resolution remote sensing image analysis. Accurate segmentation determines the success or failure of subsequent remote sensing image processing. Image segmentation is that the images dividing into several connected regions do not overlap each other according to certain rules. The algorithms are going based on the two basic characteristics of general brightness values, namely discontinuity and similarity. The first kind of method is based on the brightness of the discontinuous changes, such as the edge of the image, etc. Other is dividing images into the similar area according to the standards set, the threshold processing, for examples, threshold processing, regional growth, region separation and aggregation(Rafael C G,2002). With the enlargement of the application domain, image segmentation gradually becomes the key technology in the field of remote sensing image processing, pattern recognition and computer vision.

\section{RELATED WORKS}

Since the advantage of remote sensing image object-oriented information extraction is more and more obvious, many scholars has been done a lot of research on the issues of multiple features fusion, multi-scale and multi-temporal high resolution remote sensing image segmentation. Especially the spectrum graph theory is widely used in data dimension reduction, clustering and image 
segmentation(Zhang Tao,2009; Cui Weihong, 2010). The idea of adopting graph theory technology to image segmentation was first put forward in 1971 by Zahn(1971). He used the Minimum spanning tree of graph theory (Minimum spanning tree) for the study of clustering and image segmentation. Although the initial processing method is simplest, but it is widely used in computer vision. Since then, international developed a variety of image segmentation methods based on graph theory(Shi J, Malik J, 2000; Boykov Y, Jolly M P,2001; Grady L, 2006; Grady L, Eric L, Schwartz, 2006.). On the basis of Zahn's research, Felzenszwalb(2004) combined with minimum spanning tree algorithm and region merging method, and put forward a fast minimum spanning tree method based on adaptive threshold (FH)(Ye wei, Wang Yuanjun, 2009). The method achieved segmentation by measuring and comparing the similarity and the regional differences to determine whether to merge the two regions, but it did not take the influence of other features into account. Then YeWei(2009) put forward new optimization method which combined the minimum spanning tree algorithm with Mumford Shah theory, and got the good segmentation effect, but the segmentation rate was slower. The multi-scale and multi-level segmentation method(CUI W H, ZHANG Y,2011) was proposed based on minimum spanning tree, and successfully used in high resolution remote sensing image segmentation. However region shape feature was unused in the process of region merging. Literature(Wu Zhao cong,2013) proposed a comprehensive utilization of spectrum, texture and shape information segmentation method. It could effectively improve the quality of segmentation for the relatively rich areas of the texture information comparing with software eCognition fractal network evolution algorithm (highest-resolution network evolution that FNEA) methods, but the method was not combined with the feature of edge information and context and so on. To further enhance the multi-scale remote sensing image segmentation automated and adaptive level, considering the above methods, a method of multi-scale segmentation of high resolution remote sensing images by integrating multiple features is presented.

\section{METHODS}

Although FH method can quickly segment the different nature of the regions, but it only considers the spectrum information of pixels in a particular area, and gets a coarse segmentation result. Based on the characteristics of high resolution remote sensing images, multi-scale segmentation by integrating multiple features is put forward. The technical process is shown in Figure 1:

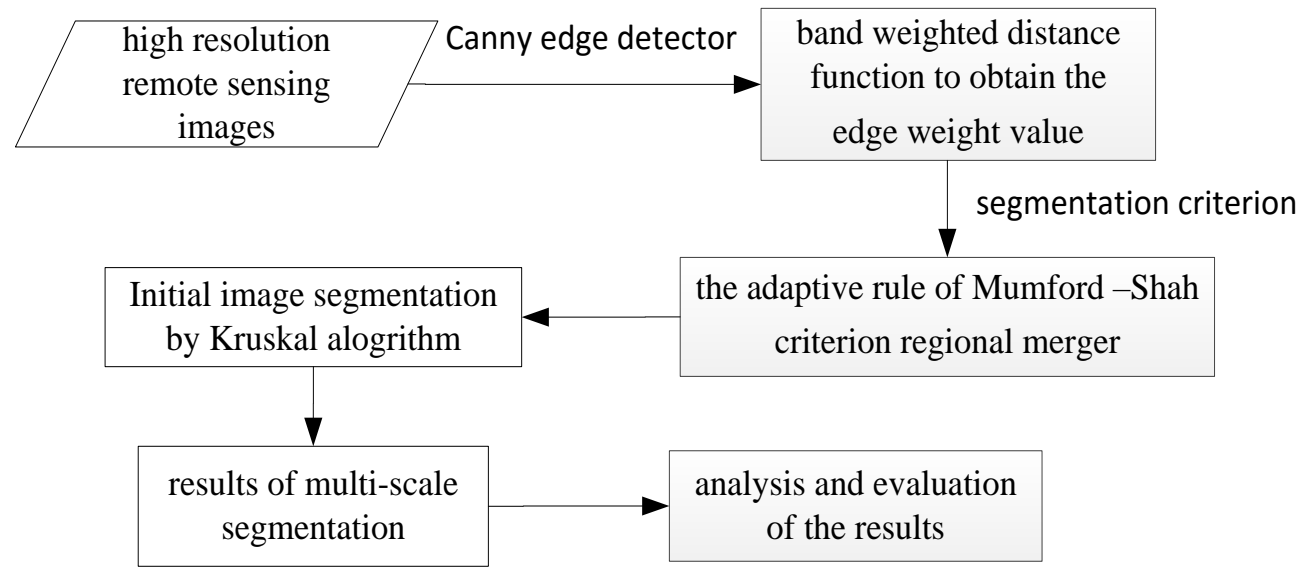

Figure 1. The technical process of multi-scale segmentation by integrating multiple features

\section{1 edge extraction by Canny operator}

The edges of the objects are the basic characteristics of image. The edge determination and extraction is very important for the entire image scene recognition and understanding and the features of image 
segmentation. The Canny operator using one-order partial derivatives of finite difference to calculate the amplitude and direction of the gradient is used widely. It is mainly modeling for brightness changes on the step-edge, which is the local extreme points of
Gaussian smoothing filter(John Canny,1986).The flow of Canny operator extracting edges is shown in Figure 2.

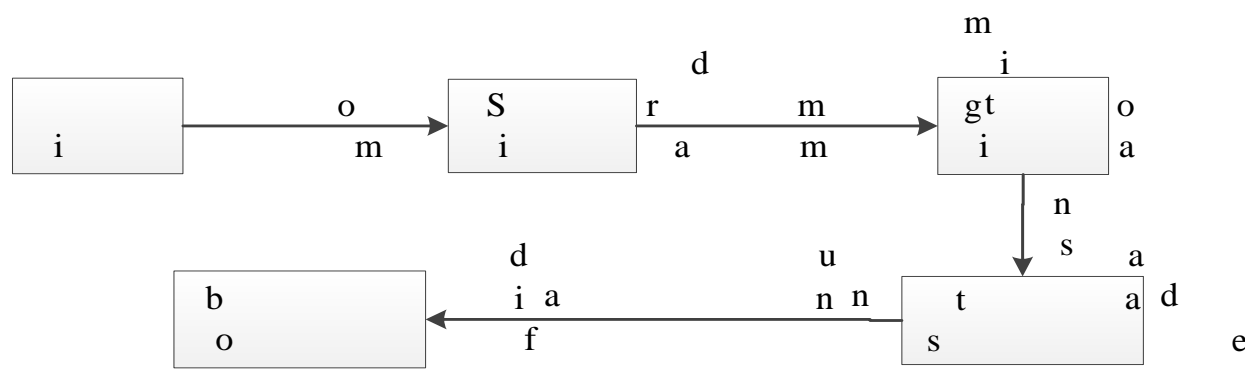

Figure 2. Canny operator edge extraction flow

According to the Canny operator definition and scale space theory, the edge detection gains $\sigma$ in scale space. It means that the Canny operator can obtain multi-scale edges by different $\sigma$.

\subsection{Band weighted distance function to obtain the edge weight value}

The image is taken as an undirected graph $G=(V, E)$ and image pixel as the vertex figure $v_{i} \in V$.Each of the adjacent edge $\left(v_{i}, v_{j}\right) \in E$ contains a corresponding weight $w\left(v_{i}, v_{j}\right)$, which is not a negative and is the pixel difference in the two vertices used to describe no similarity between two adjacent vertices. Considering the high resolution multispectral image features, if the band combination and the weights of different wave band are chosen appropriately, it can effectively distinguish the specific goal, so band weighted Euclidean distance is suited to construct the edge weights. The concrete calculating formula is generated as in equation (1):

$w(u, v)=\sum_{i=1}^{l} w_{i} d\left(u_{i}, v_{i}\right)=\sqrt{\sum_{i=1}^{l} w_{i}\left|u_{i}-v_{i}\right|^{2}}$

Where $w(u, v)$ is connecting adjacent vertices (pixels) $u$ and the edge $v$ weights, $l$ is the band number, $w_{i}$ is distance weight for band $i, \sum_{i=1}^{l} w_{i}=1, u_{i}$ is the grey value $u$ of the band $i$.

In the process of object-oriented segmentation, the edge of the band combination has better consistency and larger differences between regions each other. The standard deviation of each band is selected to calculate band weights. Each band weight calculation formula is equation (2):

$$
w_{i}=\sigma_{i} / \sum_{i=1}^{l} \sigma_{i}
$$

Where $w_{i}=$ weight of the band

$$
\begin{aligned}
& l=\text { number of the band } \\
& \sigma_{i}=\text { standard deviation of a band }
\end{aligned}
$$

We add the edge information and conduct image smoothing when structuring the edge weight graph. That means to establish scale space image and choose the corresponding edge detection scale to build the graph model. Edge weights are calculated as in equation (3):

$$
w(u, v)= \begin{cases}0 & W(u, v)=1 \\ \sum_{i=1}^{l} w_{i} d\left(u_{i}, v_{i}\right)=\sqrt{\sum_{i=1}^{l} w_{i}\left|u_{i}-v_{i}\right|^{2}} & W(u, v) \neq 1\end{cases}
$$

where $w(u, v)=$ result of edge detection .

\subsection{Segmentation algorithm based on minimum spanning tree}

It is on the basis of image graph model that Segmentation algorithm based on minimum spanning tree adopts the bottom-up (merger) strategy to generate multiple minimum spanning tree according to certain principles. The clustering (divided) algorithm can be described as: 
(1) An edge is linked together by using adjacency between two pixels usually called four neighborhood or eight neighborhood. Edge weights are calculated by the dissimilarity measure of four vertices corresponding eigenvector. The graph model are built by combining these elements.

(2) With the Kruskal algorithm, the edges are sorted in Non-decreasing order of weights, and construct the minimum spanning tree from the edge with minimum weight.

(3) If the current edge weights meet the segmentation criteria, it is added to spanning tree, otherwise not.

(4) Repeating step (3) until the current edge weight value is greater than the set value.

Segmentation is growing process of the edge of the tree from the bottom up as each spanning tree corresponding to the image in the area. The growth of the edge merge and stop criterion is the key to segmentation. The paper uses the method proposed by Felzenszwalb(2004).

\subsection{The adaptive region merging with texture information}

After the initial segmentation result is obtained by minimum spanning tree segmentation method, we count the spectrum, texture, shape and other characteristics and define region adjacency graph by region edge weight function which is designed to look region as vertex. Region edge weight function as the measurement of feature difference is also the cost function of region merging. Therefore we take the feature fusion that is the best regional feature differences into a weight function to make the minimum edge weight in the same type adjacent region.

Mumford and Shah have put forward Mumford-Shah model to represent the segmentation, and transform the optimal segmentation into minimize Mumford Shah - energy function (namely the minimum fitting error). Koepfler has simplified the Mumford Shah model as in equation (4):

$$
\begin{aligned}
E(u, K) & =\int_{\Omega / K}\left((u(x)-g(x))^{2} d x+\lambda \cdot l(K)\right. \\
\text { where } \Omega & =\text { region } \\
K & =\text { regional boundaries }
\end{aligned}
$$

$$
\begin{aligned}
& u(x)=\text { approximation for } g(x) \\
& l=\text { border length } \\
& \lambda=\text { parameter factor }
\end{aligned}
$$

He has proved that the energy minimization model can be achieved through the growth of region growing strategy and merging to get the merging criterion as in equation (5):

$$
E\left(K \backslash \partial\left(o_{i}, o_{j}\right)\right)-E(K)=\frac{\left|o_{i}\right|\left|\cdot o_{j}\right|}{\left|o_{i}\right|+\left|o_{j}\right|}\left\|u_{i}-u_{j}\right\|^{2}-\lambda \cdot l\left(\partial\left(o_{i}, o_{j}\right)\right)<0(5)
$$

The merging need reduce the energy of function,

$$
\begin{gathered}
\text { where } o_{i} \text { and } o_{j}=\text { region } i \text { and } j \\
\partial\left(o_{i}, o_{j}\right)=\text { two area shared border } \\
|o|=\text { area of the region }
\end{gathered}
$$

The algorithm must be determined to select different $\lambda_{i}$ values, and then start from the pixel iteration merge to get different segmentation results. Redding has put forward the improved full $\lambda$-schedule, the merging criteria for equation (6).

$$
t_{i, j}=\frac{\left|o_{i}\right| \cdot\left|o_{j}\right|}{\left|o_{i}\right|+\left|o_{j}\right|} \| u_{i}-\left.u_{j}\right|^{2} / l\left(\partial\left(o_{i}, o_{j}\right) \leq \lambda_{i}\right.
$$

The region merging use $\lambda_{i}$ as a scale parameter and different $\lambda$ gain the multi-scale segmentation results based on the most applicable principles.

Considering the complexity of terrain and the richness of texture information in the high resolution remote sensing images, we put forward the improvement scheme which add texture heterogeneity in Mumford-Shah measure simplified model, and conduct adaptive weighted heterogeneity of spectrum and texture information. Multi-scale rotation invariant LBP histogram $\mathrm{H}$ represent texture. We calculate weighted coefficient through regional distribution of standard deviation, then the region merging criteria can be rewritten as in equation (7).

$T=\frac{\frac{\left|o_{i}\right| \cdot\left|o_{j}\right|}{\left|o_{i}\right|+\left|o_{j}\right|}\left(w_{\text {color }}\left\|u_{i}-u_{j}\right\|^{2}+w_{\text {texture }} \cdot \chi^{2}\left(H_{i}-H_{j}\right)\right)}{l\left(\partial\left(o_{i}, o_{j}\right)\right.}$

$\chi^{2}\left(H_{i}-H_{j}\right)$ represents that the distance $\chi^{2}$ calculates texture histogram distance. Spectrum and texture 
weight coefficient $w_{\text {color }}$ and $w_{\text {texture }}$ are determined by the self-adaption as in equation (8).

$$
w_{\text {color }}=\max \left(\sigma_{1}, \sigma_{2}\right), w_{\text {texture }}=1-\max \left(\sigma_{1}, \sigma_{2}\right)
$$
$\sigma_{1}, \sigma_{2}$ are the two area objects normalized gray standard deviation .

\subsection{Evaluation}

Segmentation quality evaluation is used to adopting qualitative analysis of segmentation results, but the artificial method accuracy is affected relatively largely by subjective factors. Therefore the quantitatively and objectively evaluation of segmentation results is very important. In this paper, the efficiency and accuracy is dealt with time-consuming and Rand index.

Rand Index indicates the uniformity of the clustering results and the original data set of sample distribution. Presently using for segmentation accuracy evaluation is completely possible. For a given set of points $X=\left\{x_{1}, x_{2}, \ldots, x_{n}\right\} \quad, \quad U^{(s)}=\left\{u_{1}^{(s)}, u_{2}^{(s)}, \ldots, u_{R}^{(s)}\right\}$ and $U=\left\{u_{1}, u_{2}, \ldots, u_{T}\right\}$ are the division of two different data sets. For $1 \leq i \neq i^{\prime} \leq R, 1 \leq j \neq j^{\prime} \leq T$, there is $\bigcup_{i=1}^{R} u_{i}^{(s)}=E=\bigcup_{j=1}^{T} u_{j}, u_{i}^{(s)} \cap u_{i^{\prime}}^{(s)}=\phi=u_{i} \cap u_{i^{\prime}}$. If $U^{(s)}$ is the reference data, and $U$ is the clustering result, $m$ are the number of points in $U^{(s)}$ and $U$ appeared in the same objects. $n$ are the number of points in the $U^{(s)}$ presented in the same objects, but in $U$ appeared in a different number. $b$ are the number of points in $U^{(s)}$ appeared in a different objects, but in $U$ appeared in the same objects. $d$ are the number of points in $U^{(s)}$ and $U$ both appeared in the number of different objects. The Rand Index is

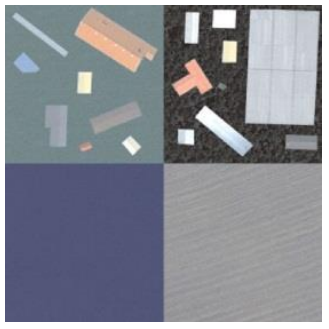

(a) Analog image calculated by the following formula as in equation (9).

$$
\text { RandIndex }=\frac{m+n}{m+n+b+d}
$$

Obviously, RanIndex values are between $[0,1]$.

When both the conditions are entirely consistent with each other, RanIndex is 1.

\section{THE EXOERIMENTAL RESULTS AND ANALYSIS}

In order to verify the validity of the method in this paper, the experiments adopt analog images and high resolution remote sensing images of radiation correction and geometry correction. A better segmentation results can get by the following parameters under the same scale parameter through a number of experiments: edge double threshold can be $[28,70]$, and $\omega_{\text {color }}=0.9, \omega_{\text {compact }}=0.5$.

\subsection{Analog image Multi-scale segmentation results and analysis}

Analog image data are image samples extracted by the combination of remote sensing images. The image size is $512 \times 512$ pixels, where different colors represent different ground objects, and the four regions have different textures, shown in Figure 3. A lot of experiments have been proceeded to set the same parameters for software eCognition and algorithm depending on the different scales, the results shown in Figure 4. The accuracy of the results obtained by statistics for the quantitative evaluation is shown in Table 1.

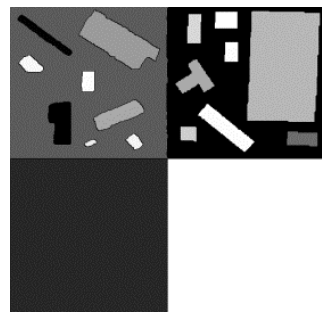

(b) Reference image

Figure 3. Analog image and reference image 


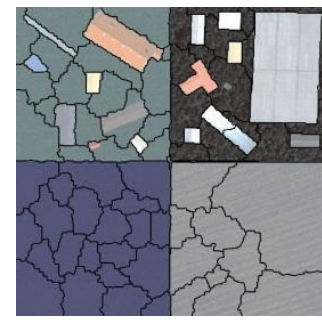

(a) Software eCognition

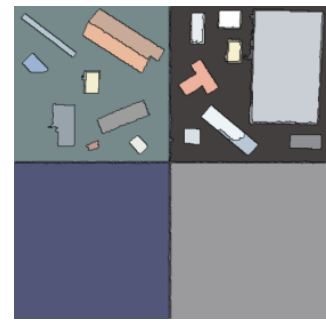

(b) this method

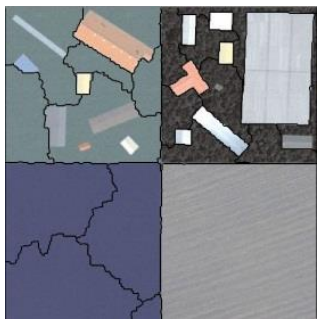

(c) Software eCognition

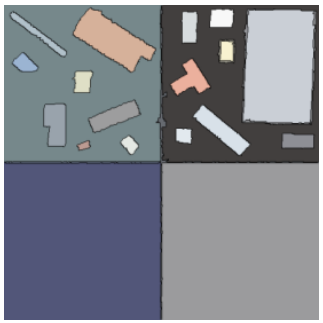

(d) this method

Scale $=50$

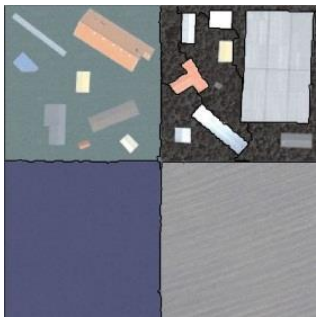

(e) Software eCognition

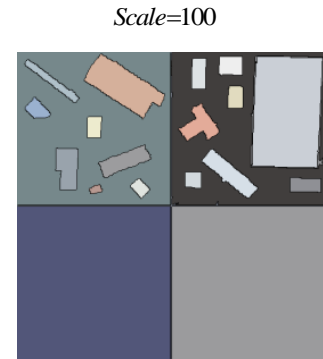

(f) this method

Scale $=150$

Figure 4. Segmentation results of the analog image

\begin{tabular}{c|c|c|c|c}
\hline Scale & & 50 & 100 & 150 \\
\hline \multirow{2}{*}{ consuming time(/s) } & Software eCognition & 1.279 & 1.186 & 1.170 \\
\cline { 2 - 5 } & this method & 1.467 & 1.591 & 1.685 \\
\hline \multirow{2}{*}{ Rand Index } & Software eCognition & 0.411 & 0.497 & 0.396 \\
\cline { 2 - 5 } & this method & 0.966 & 0.977 & 0.995 \\
\hline
\end{tabular}

Table 1. The quantitative assessment of the segmentation results of analog image

Segmentation results can be seen from Figure 4, there are obvious error segmentations at each scale in the software eCognition. In a smaller scale, over-segmentation phenomenon exists, and the texture and edge information is not sensitive, such as the lower part of the same object, but this method does not have the problem substantially because of the addition texture information; with the scale increasing, there is under-segmentation phenomenon where the upper left corner is not split out, however this method has carried around a good segmentation in a result of the edge constraint. Table 1 from a quantitative analysis can be drawn: although this method is not as efficient as segmentation software eCognition, but Rand Index is significantly higher than software eCognition, thus segmentation results are greatly improved.

\subsection{ZY-3 satellite image multi-scale segmentation results and analysis}

Analog image segmentation is not sufficiently proving the efficiency of this method, so confirmatory experiments use a resolution of $4 \mathrm{~m}$ real ZY-3 satellite image and the reference image (Figure 5). The image size is $986 \times 596$ pixels, where the figure has houses, roads, bare land and other ground objects. Multiple sets of experiments are conducted to obtain the results shown in Figure 6, the results of the quantitative analysis shown in Table 2. 


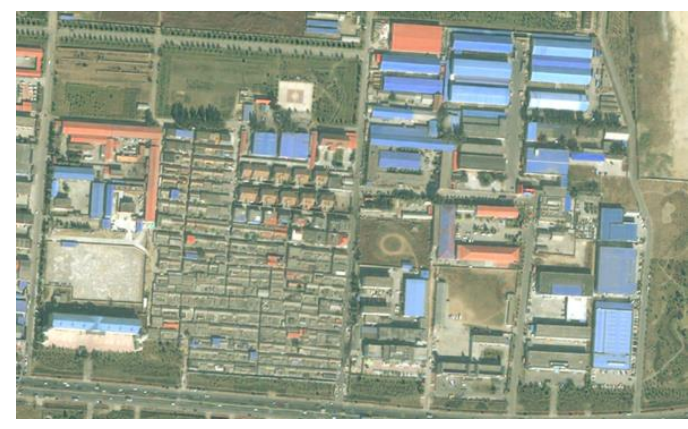

(a) ZY-3 satellite image

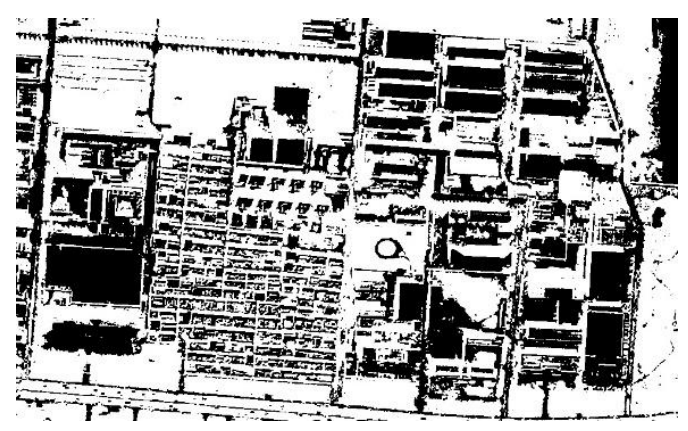

(b) Reference image

Figure 5. Analog image and reference image

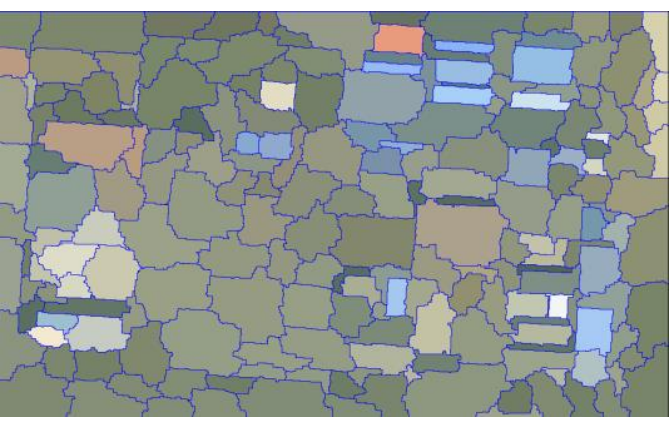

(a) Software eCognition

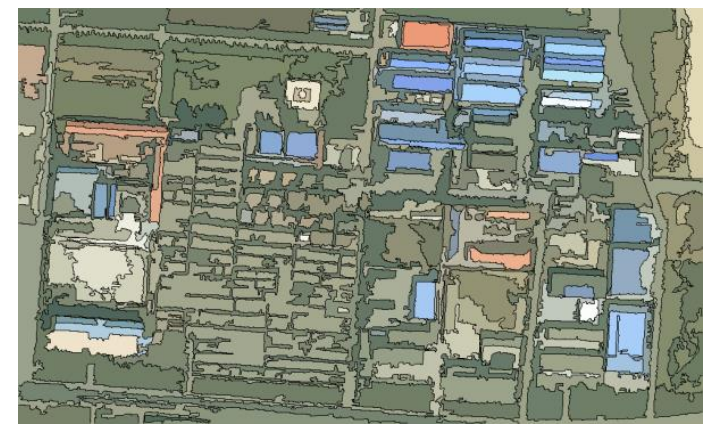

(b) this method

Scale $=50$

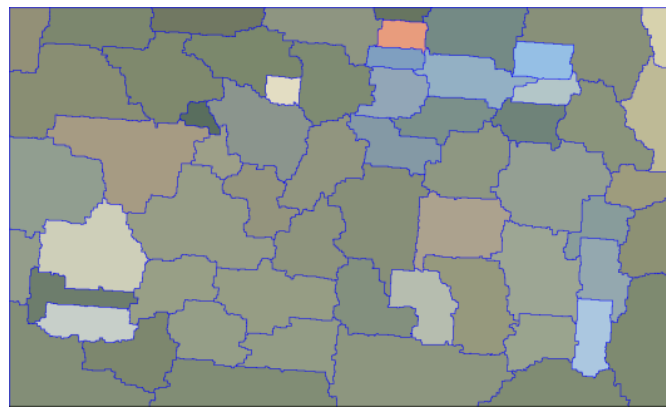

(c) Software eCognition

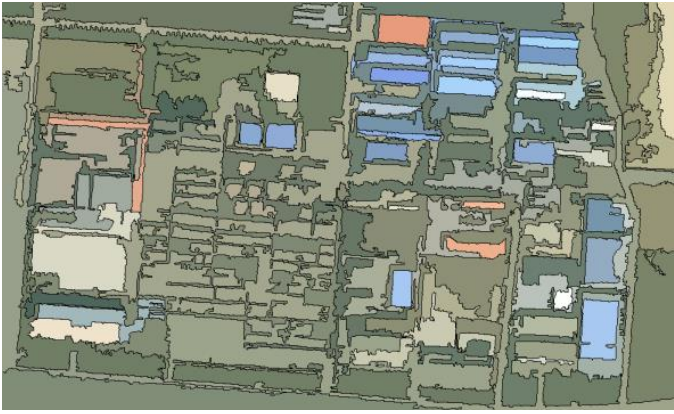

(d) this method

Scale $=100$

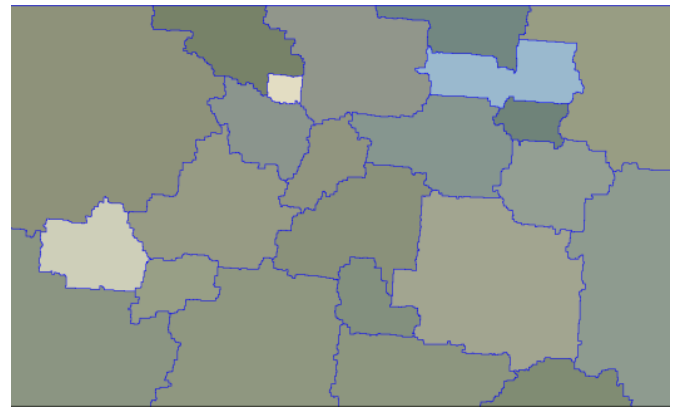

(e) Software eCognition

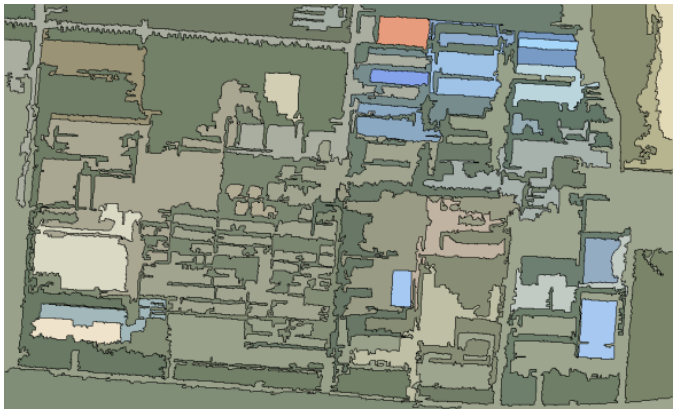

(f) this method

Scale $=150$

Figure 6. Segmentation results of the ZY-3 satellite image 


\begin{tabular}{c|c|c|c|c}
\hline Scale & & 50 & 100 & 150 \\
\hline \multirow{2}{*}{ consuming time(/s) } & Software eCognition & 3.151 & 3.027 & 2.995 \\
\cline { 2 - 5 } & this method & 4.449 & 4.184 & 3.558 \\
\hline \multirow{2}{*}{ Rand Index } & Software eCognition & 0.437 & 0.356 & 0.261 \\
\cline { 2 - 5 } & this method & 0.802 & 0.867 & 0.734 \\
\hline
\end{tabular}

Table 2. The quantitative assessment of the segmentation results of ZY-3 satellite image

Segmentation results can be obtained from Figure 6 ,

when dealing with relatively crushing ground objects, error segmentation from software eCognition is very obvious, especially roads and other objects needing edge information, and other fields with a textured ground objects. From Table 2, Quantitative analysis can be drawn: Rand Index of this method is much higher than the software eCognition, and segmentation effect is clearly better. Because of obvious over-segmentation and under- segmentation in the software eCognition, this method is well integrated various features to limit its segmentation errors. Especially through the strategy of adaptive Mumford-Shah model region merging by integrating texture features, it is reduced in the multi-scale segmentation errors and the dependence of the scale parameters. With the image size increasing, the segmentation efficiency of this method is a little lower than software eCognition, but these problems can be optimized, either through hardware GPU acceleration or improving processing speed of the algorithm.

\section{CONCLUSIONS}

Based on the use of the layers information of high-resolution images, we propose a method of multi-scale segmentation of high resolution remote sensing images by integrating multiple features. The method extracts edge information by canny operator, and constructs from the function to obtain a weighted band edge weight value, according to certain segmentation criteria, then obtains an image of the original objects using Kruskal minimum spanning tree algorithm. Last simplified Mumford -Shah model is used to merge regions by spectral information and texture information to get the segmentation results. The method is not only adopting the edge, texture and other information of high resolution images to improve the accuracy of multi-scale segmentation, but also having on adaptive Mumford-Shah criteria region merging to avoid the uncertainty of artificial setting parameters. After a lot of experiments, the proposed algorithm has better segmentation effect than FNEA algorithm of software eCognition. The method combined with a variety of features for image segmentation, but is not yet implemented fully automatic segmentation feature fusion. We will focus on unsupervised segmentation method of high resolution remote sensing images by integrating multiple features, in order to obtain the high segmentation accuracy, fast speed of operation and automatic segmentation.

\section{REFERENCES}

Rafael C G, Richard E W, 2002. Digital Image Processing $[\mathrm{M}]$. Second Edition. New Jersey: Prentice Hall.

Zhang Tao, Hong Wenxue, 2009. Texture image analysis based on graph theory $[\mathrm{J}]$. photon technology, 35(6):825-831.

Cui Weihong, 2010. Research on Graph Theory Based Object oriented High Resolution Image Segmentation[D]. Dissertation for the Ph.D of wuhan University, P.R.China.

Zahn,C.T, 1971. Graph-Theoretical Methods for Detecting and Describing Gestalt Clusters[J]. IEEE Transactions on Computers., 20(1):P.68-86.

Shi J, Malik J, 2000. Normalized cuts and image segmentation[J]. IEEE Transactions on Pattern Analysis and Machine Intelligence. 2(8): 888-905.

Boykov Y, Jolly M P,2001. Interactive graph cuts for optimal boundary and region segmentation of objects in $\mathrm{n}$-d images[C]. IEEE International Conference on Computer Vision. 105-112.

Grady L, 2006. Random walks for image segmentation[J]. IEEE Transactions on Pattern Analysis and Machine Intelligence, 28(11): 1768-1783.

Grady L, Eric L, Schwartz, 2006. Isoperimetric graph partitioning for data clustering and image 
segmentation[J]. IEEE Transactions on Pattern

Analysis and Machine Intelligence. 28(3): 469-475.

FELZENSZWALBPF, HUTTENLOCHERDP,2004.

Efficient Graph-based Image Segmentation [J]. International Journal of Computer Vision. 59(2):167-181.

Ye wei, Wang Yuanjun, 2009. Minimum Spanning Tree Image Segmentation Method Based on Mumford-Shah Model[J]. Journal of Computer-Aided Design \& Computer Graphics, 21(8):1127-1134.

CUI W H, ZHANG Y, 2011.An Effective Graph-based Hierarchy Image Segmentation[J]. Intelligent Automation \& Soft Computing, 17(7):969-981.

Wu Zhao cong, HU Zhongwen, ZhANG qian, et al. 2013. On combining spectrum、texture and shape features for Remote sensing image segmentation[J]. Journal of surveying and mapping, 42(1):44-50.

John Canny, 1986. A Computational Approach to Edge Detection [J]. IEEE Trans. Pattern Analysis and Machine Intelligence (S0162-8828), 8(6):679-698. 